BULLETIN OF THE

AMERICAN MATHEMATICAL SOCIETY

Volume 81, Number 1, January 1975

\title{
RELATIVE COMPLETIONS OF $A$-SEGAL ALGEBRAS
}

\author{
BY JAMES T. BURNHAM
}

Communicated by Richard R. Goldberg, July 14, 1974

We announce some new results about multipliers and ideal theory of $A$ Segal algebras and their relative completions. Complete details are to appear elsewhere [3], [4]. The results about multipliers (Theorem 6) represent work done jointly with Richard R. Goldberg [4].

Defintions. If $A$ is a Banach algebra, we say the subalgebra $B \subseteq A$ is an $A$-Segal algebra provided $B$ is a dense left ideal of $A, B$ is a Banach algebra with respect to a norm \|\|$_{B}$, the injection of $B$ into $A$ is continuous, and multiplication is (jointly) continuous on $A \times B$ into $B$. We shall always suppose that $A$ does not have an identity.

The relative completion of $B$ with respect to $A$, denoted $\widetilde{B}^{A}$, is defined by

$$
\widetilde{B}^{A}=\bigcup_{n>0} \overline{S_{B}(\eta)^{A}},
$$

where $S_{B}(\eta)=\left\{f \in B \mid\|f\|_{B} \leqslant \eta\right\}$ and $\bar{E}^{A}$ is the $A$ closure of $E$. For $f \in \widetilde{B}^{A}$ we define $\|f\|$ by

$$
\|f\| \mid=\inf \left\{\delta \mid f \in \overline{S_{B}(\delta)^{A}}\right\} .
$$

THEOREM 1. If $B$ is an A-Segal algebra, then $\widetilde{B}^{A}$ (with norm || $\mid$ II) is an A-Segal algebra. Furthermore, if $B$ has right approximate units which are bounded in the A-norm, then $B$ is a closed left ideal of $\widetilde{B}^{A}$ and the embedding of $B$ into $\widetilde{B}^{A}$ is isometric if the approximate units have A-norm one.

In case $A$ and $B$ share common right approximate units of $A$-norm one, then $\widetilde{B}^{A}$ has a rather simple description which permits a straightforward proof of all of the assertions of Theorem 1. Such is the case when $A=$ $L^{1}(G)$ and $B$ is an ordinary Segal algebra [6, p. 16]. Indeed,

THEOREM 2. With $A$ and $B$ as in the preceding paragraph and $U$ denoting (a set of) common right approximate units, we have 


$$
f \in \widetilde{B}^{A} \Leftrightarrow M \equiv \operatorname{Sup}\left\{\|u * f\|_{B} \mid u \in U\right\}<\infty,
$$

and in this case $\|f\|=M$.

From here on we suppose that $A$ and $B$ have common right approximate units of $A$-norm one. The following theorem, which has the assertion of the second sentence in Theorem 1 as a consequence, is of independent interest.

THEOREM 3. $S_{B}(\delta)={\overline{S_{B}(\delta)}}^{A} \cap B$; in particular, if $B=\widetilde{B}^{A}$, then $S_{B}(\delta)=\overline{S_{B}(\delta)^{A}}$.

Definition 4. We say $B$ is singular provided $B \neq \widetilde{B}^{A}$.

Perhaps the simplest example of a singular $A$-Segal algebra and its relative completion is the pair $\left(C(G), L^{\infty}(G)\right)$, where $G$ is an infinite compact group and $A=L^{1}$. Additional examples of singular Segal algebras are given in [3] and [4]; a more detailed analysis of singularity is given in [3].

Some results which are useful for an analysis of multipliers and the ideal theory of $A$-Segal algebras and their relative completions are summarized in

THEOREM 5. (1) If $B$ is a closed ideal in the A-Segal algebra $B_{1}$, then $B_{1} \subseteq \widetilde{B}^{A}$. Let $U$ denote right approximate units for $B$. (2) If $f \in \widetilde{B^{A}}$, then $f \in B \Leftrightarrow$ given any $\epsilon>0$ there exists $u(f, \epsilon) \equiv u \in U$ so that $\|u f-f\| \leqslant \epsilon$. (3) $A \widetilde{B}^{A} \subseteq B$ and, hence, $\widetilde{B}^{A} \cdot \widetilde{B}^{A} \subseteq B$. We thus see that $\widetilde{B}^{A}$ fails to factor if $B$ is singular.

We now specialize to the case where $A=L^{1}(G)$, and $B=S(G)$ is a symmetric Segal algebra as defined by $H$. Reiter [6, p. 17]. Here, $G$ denotes a locally compact nondiscrete group. The (multiplier) algebra of bounded linear operators from $L^{1}(G)$ into $S(G)\left(\widetilde{S}^{L^{1}}(G)\right)$ for which $T(f * g)=f * T g$ is denoted $\left(L^{1}, S\right)\left(\left(L^{1}, \widetilde{S}^{L^{1}}\right)\right)$.

THEOREM 6. Let $\left\langle e_{\alpha}\right\rangle$ be a bounded approximate identity for $L^{1}(G)$. For a measure $\mu \in M(G)$ the following three conditions are equivalent:

(1) $\operatorname{Sup}_{\alpha}\left\|e_{\alpha} * \mu\right\|_{S}<\infty$; (2) $\mu \in\left(L^{1}, S\right)$; (3) $\mu \in\left(L^{1}, \widetilde{S}^{L^{1}}\right)$.

Furthermore, if $\left(L^{1}, S\right) \subseteq L^{1}(G)$, then $\left(L^{1}, S\right)$ is isometrically isomorphic with $\widetilde{S}^{L}{ }^{1}$.

For our final theorems we require that $G$ be an infinite compact group. All unexplained notation may be identified from the analogous results in [5].

TheOREM 7 [5, 38.9, p. 453]. Let $S(G)$ be a singular Segal algebra. Let 
$P$ be any subset of $\Sigma$. Let $F$ be a closed linear subspace of $\widetilde{S}^{L 1}(G)$ for which $F \cap S(G)=S_{p}(G)$ and $F \subset \widetilde{S}_{p}^{L^{1}}(G)$. Then $F$ is a closed two-sided ideal in $\widetilde{S}^{L^{1}}(G)$; conversely, all closed two-sided ideals in $\widetilde{S}^{L^{1}}(G)$ have this form. Furthermore, the quotient algebra $\widetilde{S}^{L^{1}}(G) / S(G)$ is a zero algebra. The closed two-sided ideals in $\widetilde{S}^{L^{1}}(G)$ for which the quotient algebra is a zero algebra are exactly the closed linear subspaces of $\widetilde{S}^{L^{1}}(G)$ that contain $S(G)$.

THEOREM 8. Suppose $S(G)$ is a singular Segal algebra. For each $\sigma \in \Sigma$, $\tilde{S}_{\{\sigma\}}^{L 1}(G)$ is a regular maximal proper two-sided ideal in $\widetilde{S}^{L^{1}}(G)$. If $M$ is a nonzero bounded linear functional on $\widetilde{S}^{L^{1}}(G)$ which vanishes on $S(G)$, then $M^{-1}(0)$ is a closed maximal proper two-sided ideal in $\widetilde{S}^{L^{1}}(G)$ for which $\tilde{S}_{\{\sigma\}}^{L 1}(G) / M^{-1}(0)$ is a 1 dimensional zero algebra. Every maximal closed proper two-sided ideal of $\widetilde{S}^{L^{1}}(G)$ not of the form $\widetilde{S}_{\{\sigma\}}^{L^{1}}(G)$ is obtained in this way.

For the ideal theory of $A$-Segal algebras with approximate identities, we refer to [1] and [2].

\section{REFERENCES}

1. James T. Burnham, Closed ideals in subalgebras of Banach algebras. I, Proc. Amer. Math. Soc. 32 (1972), 551-555. MR 45 \#146.

2. Closed ideals in subalgebras of Banach algebras, Monatsh. Math. 78 (1974), 1 -3.

3. - The relative completion of an A-Segal algebra is closed, Proc. Amer. Math. Soc. (to appear).

4. James T. Burnham and Richard R. Goldberg, Multipliers of $L^{1}(G)$ into Segal algebras (to appear).

5. E. Hewitt and K. A. Ross, Abstract harmonic analysis. Vol. II: Structure and analysis for compact groups, Die Grundlehren der math. Wissenschaften, Band 152 , SpringerVerlag, New York and Berlin, 1970. MR 41 \#7378; erratum, 42, p. 1825.

6. Hans Reiter, $L^{1}$-algebras and Segal algebras, Lecture Notes in Math., vol. 231, Springer-Verlag, Berlin and New York, 1971.

DEPARTMENT OF MATHEMATICS, OKLAHOMA STATE UNIVERSITY, STILLWATER, OKLAHOMA 74074 